normal (within $10 \%$ of normal GFR if previously abnormal) GFR. Partial Response (PR) was defined as $a \geq 50 \%$ reduction in proteinuria to subnephrotic levels and normal or near-normal GFR. Renal outcomes were assed at one year post biopsy.

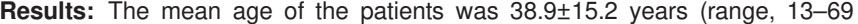
years). The study included 85 females $(87.6 \%)$ and 12 males (12.4\%). The clinical presentations were nephrotic syndrome, nephritic syndrome, and asymptomatic urinary abnormalitiesin 38 (39.2\%), $20(20.6 \%), 39(40.2 \%)$ patients, respectively. Nine patients were classified Class III $(9.3 \%$, including 2 as ClassIII $+\mathrm{V}), 82$ as Class IV (84.5\%, 10 as Class IV-segmental(IV-S) (10.3\%) and 72 as Class IVglobal (IV-G) (74.2\%), including 4 as Class IV-G+V) and 6 as Class V (6.2\%). Forty-two(43\%) patients presented with acute and $55(57 \%)$ with features of chronic TMA. All patients had received treatment with standard immunosuppressants (55\% mycophenolate, 39\% cyclophosphamide, $6 \%$ other regimen) and steroids.

Abstract OP0201 - Table 1

\begin{tabular}{|l|l|}
\hline Acute TMA features & Chronic TMA features \\
\hline -Endothelial swelling with partial or & -Capillary wall thickening with double \\
complete occlusion of lumina; & contours; \\
- Microthrombi, focal or global; & -Organizing capillary thrombi; \\
fragmented RBC on glomerular & -Glomerularischemic collapse with \\
subendothelial space and/or & afferent arteriolar occlusion; \\
mesangial areas; & -Segmental/global glomerulosclerosis \\
-Mesangiolysis, focal, & \\
segmental/global; & \\
-Glomerularcongestion with efferent & \\
arteriolarocclusion & \\
\hline
\end{tabular}

At 12 months, CR was observed in 37 patients (38.1\%), PR in $22(22.6 \%)$ and no response in $38(39.1 \%)$. Sixty-one patients $(62.9 \%)$ were antiphospholipid positive $(\mathrm{aPL})$ and $37(38.1 \%)$ received anticoagulation with vitamin-K antagonist (VKA) and/or heparins. Presence of aPLs(OR, 2.4; 95\% confidence interval-Cl-, $1.2-$ 7.3; $\mathrm{p}=0.03)$, anti-DNA positivity (OR, $12.8 ; 95 \% \mathrm{Cl}: 3.0$ to $71.3 ; \mathrm{p}=0.002)$, and chronic features of TMA (OR, 3.0; $95 \% \mathrm{Cl}: 1.2$ to $17.5 ; \mathrm{p}=0.04)$ were all found to be associated with no response. When limiting the analysis to aPL positive patients, after adjusting for type of immunosuppressant therapy and LN class on biopsy, variables that were significantly associated with $\mathrm{CR}+\mathrm{PR}$ were features of acute TMA rather than chronic (OR, 8.62; 95\% Cl: 1.4 to $97.1 ; \mathrm{p}=0.03)$ and the use of VKA/heparins(OR, 2.1; 95\% Cl, 1.02-16.2; $\mathrm{p}=0.046)$

Conclusions: In patients with concomitant LN and TMA, the presence of aPL and chronic features of TMA were associated with poorer renal outcomes. In patients with $\mathrm{aPL}$, the use of anticoagulation appeared protective and warrants further investigation as a therapeutic tool, especially in the setting of acute TMA. Disclosure of Interest: None declared DOI: 10.1136/annrheumdis-2018-eular.6216

\section{OP0202 GENE EXPRESSION PROFILES IN PRIMARY SJÖGREN'S SYNDROME WITH AND WITHOUT SYSTEMIC MANIFESTATIONS}

C. Vitali ${ }^{1}$, M. Dolcino ${ }^{2}$, R. Andracco ${ }^{3}$, A. Pelosi ${ }^{4}$, P. Fiore ${ }^{4}$, W. Maglione ${ }^{3}$ E. Zaccara ${ }^{3}$, N. Del Papa ${ }^{3}$, A. Puccetti ${ }^{4}$. ${ }^{1}$ Dept. Rheumatology, San Giuseppe Institute, Como; ${ }^{2}$ Dept. of Medicine, University of Verona, Verona; ${ }^{3}$ Dept. Rheumatology, ASST G. Pini-CTO, Milano; ${ }^{4}$ Immunology Area, Bambino Gesù Hospital, Rome, Italy

Background: Different phenotypes characterise the clinical spectrum of primary Sjögren's syndrome (SjS). Patients with a clinical expression limited to glandular features (GFs) are classically distinguished from patients with extra-glandular manifestations (EGMs). The former patients often complain higher level of fatigue and widespread pain (WP). (Segal et al. 2013) This suggests that gene expression pattern may be different in the two subgroups.
Objectives: To investigate the differences of gene expression in SjS patients with GFs and in those with EGMs.

Methods: Nineteen patients with SjS were selected for the study. Gene expres sion in peripheral blood mononuclear cells (PBMCs) was analysed in 4 patients with EGMs and 4 patients with GFs alone using Clariom D human Affymetrix gene chip (Affymetrix, Santa Clara, CA, USA), and compared to that found in healthy controls. Differences in gene expression were evaluated by analysis of variance (ANOVA) and Step-Up FDR-controlling procedure, being FDR corrected $p$ value $\leq 0.01$ and fold change $>2$ considered as statistically significant.

Validation of the gene overexpression was performed by quantitative Real Time (qRT)-PCR in PBMCs from all the selected SjS patients, using the $\Delta \Delta \mathrm{Ct}$ method for comparing relative fold expression differences.

Results: All the enrolled SjS patients (18 females and 1 male) had a positive lip biopsy, while anti-SSA/Ro antibodies were detected in 10/11 and 6/8 of the patients with EGMs and with GFs alone, respectively. ESSDAI value ranged from 7 to 55 in patients with EGMs (median 17), and from 0 to 2 in patients with GFs alone (median 1).

In both types of patients, the functional analysis of the two transcriptomes showed a large number $(>1000)$ of modulated genes that are involved in the well-known pathological processes of SjS, i.e., apoptosis, inflammatory response, immune response, type I and type II interferons, and Toll-like receptors signalling.

Genes modulated only in patient with EGMs showed a significant enrichment of the biological processes associated with immune response $(79 \%$ of all enriched processes) and, namely, of the molecular pathways related to B cell activation. The analysis of the transcripts expressed only in patients with GFs alone showed instead a preponderant enrichment in different metabolic processes (43\%) and in processes associated with the central perception of the stimuli. Indeed, genes involved in sensory perception and in nociceptive signals (i.e., ANPEP, TNRF1 P2RY1, IFNG) were modulated exclusively in patients with GFs alone. The significant differential expression of selected genes in the two SjS subgroups was confirmed by the qRT-PCR analysis.

Conclusions: These data indicate that in SjS patients with GFs alone a dysregu lation of pain pathways (namely beta-adrenergic receptor and Notch signalling may play a role in the development of WP that is common in this subset of patients. The biological mechanisms triggering the activation of these genes remain to be completely clarified.

Disclosure of Interest: None declared

DOI: 10.1136/annrheumdis-2018-eular.5119

\section{THURSDAY, 14 JUNE 2018}

\section{How monogenetic autoinflammatory diseases help to understand and treat rheumatic diseases}

\begin{tabular}{|l|l}
\hline OP0203 & SAFETY AND EFFICACY OF INTRAVENOUS \\
& ADMINISTRATION OF BONE-MARROW DERIVED \\
& MESENCHYMAL STROMAL CELLS IN THERAPY \\
& REFRACTORY JUVENILE IDIOPATHIC ARTHRITIS \\
& PATIENTS, A PHASE IB/IIA PILOT-STUDY
\end{tabular}

J.F. Swart ${ }^{1}$, S. de Roock ${ }^{1}$, R.J. Nievelstein ${ }^{2}$, I. Slaper-Cortenbach ${ }^{3}$, J.J. Boelens ${ }^{4}$ N. Wulffraat ${ }^{1} .{ }^{1}$ Pediatric Rheumatology; ${ }^{2}$ Pediatric Radiology; ${ }^{3}$ Cell Tharpy Facility; ${ }^{4}$ Pediatric Immunology, UMC UTRECHT, Utrecht, Netherlands

Objectives: To compare the total number of adverse events (AE's) before and after mesenchymal stromal cell (MSC) infusion in refractory JIA and to evaluate its effectiveness.

Methods: Single-centre Phase lb/lla, open label intervention study in JIA patients previously failing all biologicals registered for their diagnosis. Six patients will receive 2 million $/ \mathrm{kg}$ intravenous infusions of allogeneic bone-marrow derived MSC. In case of ACR-Ped30-response but subsequent loss of response one and maximal two repeated infusions are allowed.

Results: Six JIA patients with 9.2 years median disease duration, still active arthritis and damage were included. All had failed methotrexate, corticosteroids and median 5 different biologicals. MSC were administered twice in 3 patients. No acute infusion reactions were observed and a lower post-treatment than pre-treatment incidence in AE's was found. The one sJIA patient had again an evolving macrophage activation syndrome, 9 weeks after tocilizumab discontinuation and 7 weeks post- MSC infusion.

Eight weeks after one MSC infusion, 4 patients showed less active joints, 5 patients improved in many clinical parameters and inflammatory parameters decreased in $3 / 4$. After 1 year, we found significantly lower active joint counts, 
improved well-being scores, normalised median ESR- and CRP-levels. Inactive disease was reached by 3 patients at 1 year.
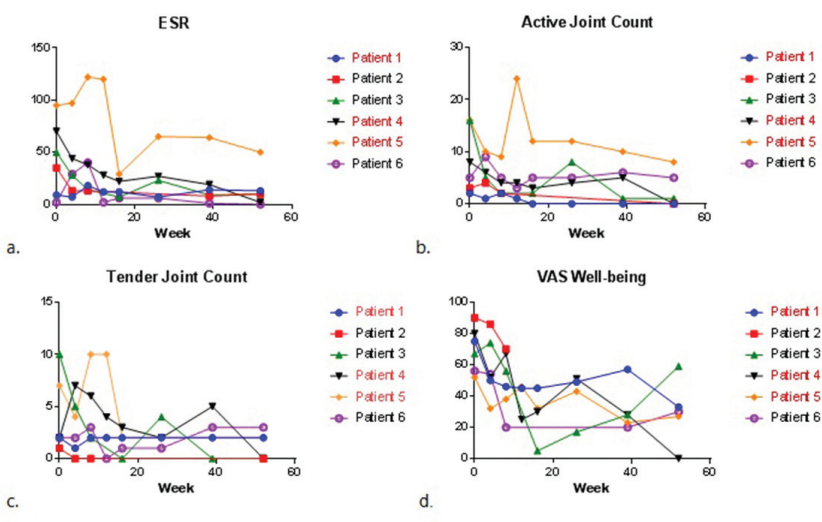

$\rightarrow$ Patiert 1

- Patiert 2

$\rightarrow$ Patiert

$\rightarrow$ Pstiert

$\rightarrow$ Patiert 6

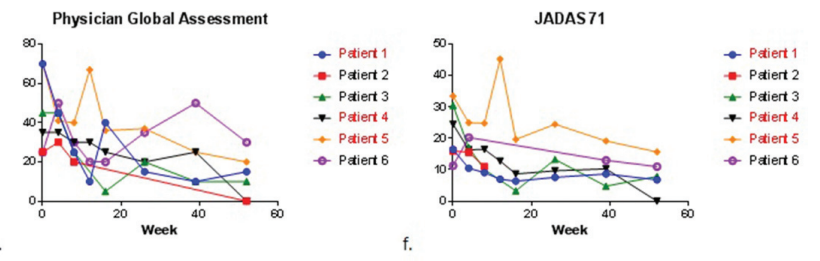

Conclusions: MSC infusions in refractory JIA patients are safe, although in SJIA stopping the 'failing' biologic treatment carries a risk of a MAS flare since the drug might still suppress the systemic features. Furthermore, intravenous administration of MSC might be efficacious even in multiple biological-failing JIA patients with damage.

Disclosure of Interest: None declared

DOI: 10.1136/annrheumdis-2018-eular.4563

\section{THURSDAY, 14 JUNE 2018}

\section{Joint EULAR - EFIS session: I've got a B in my bonnet}

\section{OP0204 DOMINANT B CELL RECEPTOR CLONES IN PERIPHERAL BLOOD PREDICT ONSET OF ARTHRITIS IN INDIVIDUALS AT RISK FOR RHEUMATOID ARTHRITIS - A VALIDATION COHORT}

A. Musters ${ }^{1}$, M.H. van Beers-Tas ${ }^{2}$, M.E. Doorenspleet ${ }^{1}$, P.L. Klarenbeek ${ }^{1}$, B.D. van Schaik $^{3}$, A.H. van Kampen ${ }^{3}$, F. Baas ${ }^{4}$, D. van Schaardenburg ${ }^{2}$, N. de Vries ${ }^{1}$. ${ }^{1}$ Clinical Immunology and Rheumatology, Amsterdam Rheumatology and Immunology Center / $A M C ;{ }^{2}$ Rheumatology, Amsterdam Rheumatology and Immunology Center / Reade; ${ }^{3}$ Clinical Epidemiology, Biostatistics and Bioinformatics; ${ }^{4}$ Genome Analysis, Academic Medical Center, Amsterdam, Netherlands

Background: A phase characterised by the presence of specific autoantibodies and arthralgia's in the absence of clinically evident synovial inflammation often precedes the onset of rheumatoid arthritis (RA). However, only a subset of these $R A$-risk individuals will develop active disease in the short term ${ }^{1}$. Recent findings show that dominant B-cell receptor (BCR) clones in peripheral blood can accurately predict imminent onset of arthritis in these $R A$-risk individuals. ${ }^{2}$

Objectives: To validate the predictive role of BCR clones in peripheral blood in $R A$-risk individuals in a larger cohort.

Methods: The BCR repertoire in peripheral blood was analysed using next-generation $\mathrm{BCR}$ sequencing in a prospective cohort study of 129 RA-risk individuals from Reade. Like earlier, BCR clones expanded beyond $0.5 \%$ of the total repertoire were labelled highly expanded clones (HECs), shortly referred to as dominant BCR clones, and individuals were labelled BCR-positive if peripheral blood at study baseline showed $\geq 5$ dominant BCR clones.

Results: We observed that the number of dominant $B C R$ clones was increased in $R A$-risk individuals who developed arthritis within 3 years, compared to RA-risk individuals who did not $10.6 \pm 5.3$ vs $2.2 \pm 2.8$ (mean $\pm S D$; $p<0.0001$ ). When creating a ROC curve we could replicate that the most optimal cut-off for this test is at $\geq 5$ dominant BCR clones in the peripheral blood (figure $1 \mathrm{~A}$ ), dividing the cohort in 45 BCR-positive individuals and 84 BCR-negative individuals. None of the BCR-clone negative individuals developed arthritis within 36 months. Within the total follow-up of 104 months only $8 \%$ of the BCR-clone negative individuals developed arthritis compared to $76 \%$ of the BCR-clone positive individuals, resulting in a relative risk of 9.1 ( $95 \% \mathrm{Cl}: 4.4$ to $18.8, p<0.0001)$.

To test whether a higher number of dominant BCR clones correlates with higher risk of arthritis BCR-clone positive individuals were subdivided into three groups: 5-9 HECs $(n=27), 10-14$ HECs $(n=13)$ and $\geq 15$ HECs $(n=5)$. The Kaplan-Meier curve for all groups is shown in figure 1B (logrank test between BCR-clone negative group and positive subgroups: $p<0.0001)$. Having 10 or more HECs corresponded with a positive predictive value of $83 \%$ and a negative predictive value of $87 \%$ within 3 years. The BCR clonality test clearly added to existing indices of RA risk in RA-risk individuals (data not shown).

A
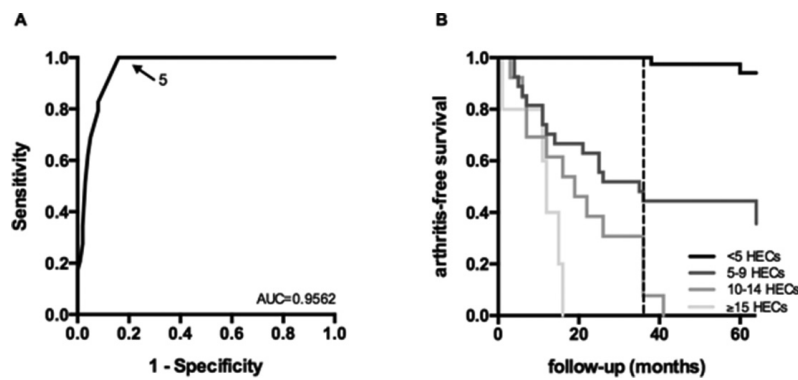

Abstract OP0204 - Figure 1 Predictive value of dominant BCR clones (A) reciver operating characteristic $(\mathrm{ROC})$ curves for the number of dominant clones, in at-risk individuals (dotted line at 36 months)

Conclusions: In this external validation cohort we could replicate the fact that dominant BCR clones in peripheral blood predict imminent onset of clinical symptoms of RA in seropositive arthralgia patients with high accuracy. Furthermore, a highly significant association correlating a higher number of dominant BCR clones with higher risk was shown. We hope these results will support evaluation of early interventions that prevent onset of arthritis.

\section{REFERENCES:}

[1] Bos WH, et al. Ann. Rheum. Dis 2010;69.

[2] Tak PP, et al. Ann. Rheum. Dis 2017;76.

Acknowledgements: Intellectual property PCT/EP2017/067047. N de Vries, PP Tak, ME Doorenspleet, PL Klarenbeek. 'METHOD FOR DETERMINING THE RISK OF DEVELOPING ARTHRITIS'.

Disclosure of Interest: A. Musters: None declared, M. van Beers-Tas: None declared, M. Doorenspleet: None declared, P. Klarenbeek: None declared, B. van Schaik: None declared, A. van Kampen: None declared, F. Baas: None declared, D. van Schaardenburg: None declared, N. de Vries Grant/research support from: IMI (ABIRISK, BeTheCure), CTMM (TRACER), LSH (MODIRA), Pfizer, Roche, Janssen, GSK, Consultant for: UCB, MSD

DOI: 10.1136/annrheumdis-2018-eular.3471

\begin{tabular}{l|l}
\hline OP0205 & APRIL INDUCES A NOVEL SUBSET OF IGA+ \\
REGULATORY B CELLS THAT SUPPRESS \\
INFLAMMATION THROUGH THE EXPRESSION OF IL-10 \\
AND PD-L1
\end{tabular}

C. Fehres ${ }^{1}$, N. van Uden ${ }^{1}$, N. Yeremenko ${ }^{1}$, L. Fernandez ${ }^{2}$, G. Franco Salinas ${ }^{1}$, L. van Duivenvoorde ${ }^{1}$, B. Huard ${ }^{3}$, J. Morel ${ }^{4}$, H. Spits ${ }^{5,6}$, M. Hahne $^{2}$, D. Baeten ${ }^{1}$ ${ }^{1}$ Clinical Immunology and Rheumatology and Experimental Immunology, Academic Medical Center, Amsterdam, Netherlands; ${ }^{2}$ Institut de Genetique Moleculaire de Montpellier, Universite de Montpellier, Montpellier, ${ }^{3}$ Institute for Advanced Biosciences, University Grenoble Alpes, Grenoble; ${ }^{4}$ Department of Rheumatology, Universite de Montpellier, Montpellier, France; ${ }^{5}$ Academic Medical Center, ${ }^{6}$ AIMM Therapeutics, Amsterdam, Netherlands

Background: Regulatory B cells (Bregs) are immunosuppressive cells that modulate immune responses through multiple mechanisms, such as the production of $\mathrm{IL}-10$ and the skewing of $\mathrm{T}$ cell differentiation in favour of a regulatory phenotype. However, the signals required for the differentiation and activation of these cells remain still poorly understood. We have already shown that overexpression of the TNF family member A PRoliferation-Inducing Ligand (APRIL) reduces the incidence and severity of collagen-induced arthritis $(\mathrm{CIA})$ in mice. 